\title{
Geo-linguistic fingerprint and the evolution of languages in Twitter
}

\author{
Eitan Altman \\ INRIA, 2004 Route des Lucioles, \\ 06902 Sophia-Antipolis Cedex, France \\ email: Eitan.Altman@inria.fr
}

\author{
Yonathan Portilla \\ LIA, UAPV, 339, chemin des Meinajaries \\ 84911 Avignon cedex 9, France \\ email: yonathan.hp@gmail.com
}

\begin{abstract}
Having access to content of messages sent by some given group of subscribers of a social network may be used to identify (and quantify) some features of that group. The feature can stand for the level of interest in some event or product, or for the popularity of some idea, or a musical hit or of a political figure. The feature can also stand for the way the written language is used and transformed, the way words are spelled and the way new grammatical rules appear. This paper has two goals. First, we identify features of groups of subscribers that have their geographic location and their language in common. We develop a methodology that allows one to perform such a study using freely available statistical tools which makes use of a part of all tweets which Twitter makes available for free over the Internet. The methodology is based on the fact that one can differentiate among some geographic areas according to the activity pattern of tweets during the time of the day. The second objective is to present our findings on the way spelling and new words have are used in Twitter. We analyze differences in appearance of new spellings among communities that are characterized by different locations but have a common language.
\end{abstract}

\section{INTRODUCTION}

Unlike many other social networks whose business model is mainly based on offering advertisements, twitter makes money by selling content: the content of a large portion of transmitted messages is sold to interested companies. One can buy almost all the content for around thirty thousand dollars a month. One can receive smaller portions for lower prices. A small portion of around $1 \%$ is made available for free. The fact that such a huge amount of messages is made available makes twitter attractive as a tool for learning about opinions in a large population. Twitter can serve as an alternative to opinion polls for market analysis not only in the context of selling goods but also for opinion trends analysis such as election campaigns [1], [2]. Twitter allows to access some information for free through different APIs (Application Program Interface).

The methodology is based on the fact that one can differentiate among some geographic areas according to the activity pattern of tweets during the time of the day. More precisely, we make use of the fact that the amount of messages generated by subscribers at a given location changes during the time of the day in a periodic way which may differ from one region to another. For example, this activity is much lower when most people in that region are asleep late at night.

We apply this methodology to the study of new spellings or of new words created in twitter messages. On the description of and reasons for this phenomenon in social media such as SMS, chats and twitter, the reader is referred to [3], [4], [5].

We note that there are other ways of obtaining geolocalisation of messages as well as the identification of language in which they are written, based on information that are available in some of tweets. The use of such information would require the user to have software tools that are not available on the Internet for free public use. We thus decided to focus in this paper on a methodology that can be widely used relying on the "trendistic" API (availble for free use on the Internet, see http://trendistic.indextank.com/).

\section{Periodograms of DAiLy ACtivity: A GEO-LINGUISTIC FINGERPRINT}

Figure 1 displays the frequency of appearance of the words "to, the, el, y, a, i" over a period of a month. The frequency of each of these words has a periodic behavior where the period corresponds to one day. We also observe that the words "To", "I" and "The" have a very similar wave form, and so do the words "El" and "Y". The word "A" has a distinct wave form differenet from the other two. The first group contains words that are frequently used in English, where as the necond group corresponds to words that appear frequently in Spanish. The word "A" appears frequently in many languages (e.g. English, Spanish, French). The word $Y$ appears also in French but its frequency there is much smaller. We conclude that words that are typical to one specific language have a common pattern, which we call a "fingerprint" of the language.

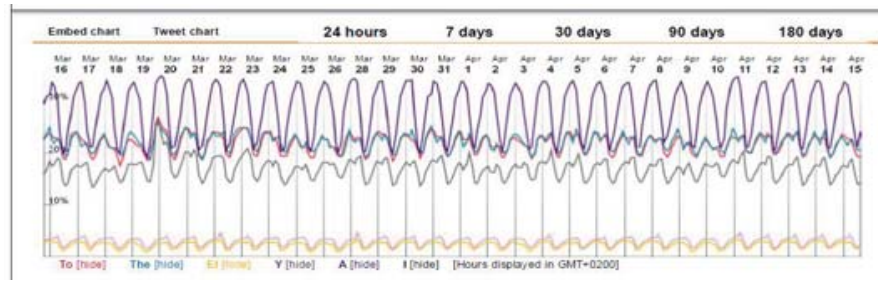

Fig. 1. The frequency of appearance of the words "to, the, el, y, a, i"

Figure 2 presents a typical german finger print. The same daily period is seen to be common to three different words that are very common in German.

Next we give an example of several spanish words, see Figure 3. There are two exceptions. We included an English word, "the", which is the most popular word in the figure. It 


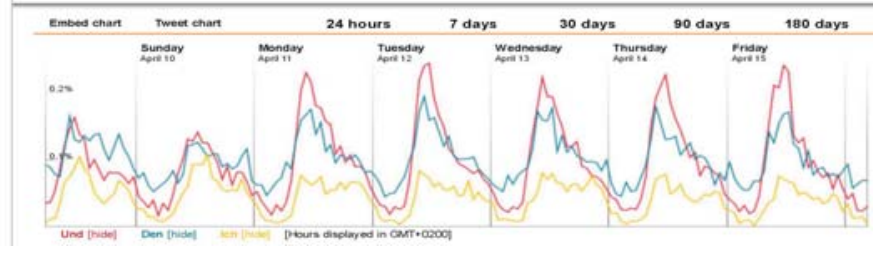

Fig. 2. The frequency of appearance of several german words during 7 days. A very clear common periodic daily pattern appears.

appeared in around $9 \%$ of all tweets. It indeed has a completely different periodic pattern. The second exception is the word "La" which frequently appears not only in Spanish but also in French and Italian. Nevnerthless, unlike "and", we observe much reseemblence to the pattern of Spanish words. A possible explanation could be that there are significantly more tweets in Spanish than in French and Italian. Therefore the periodogram of "La" is closer to the spanish even if spanish and french words had quite different periods.

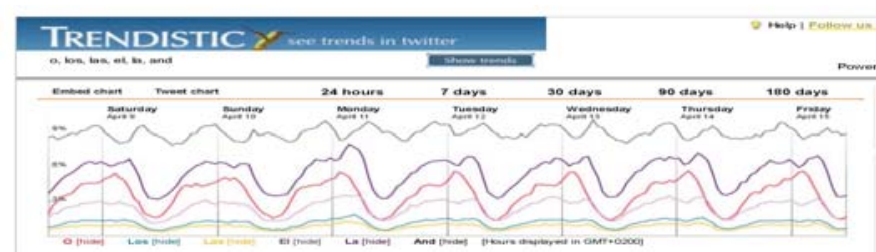

Fig. 3. The frequency of appearance of several spanish words during 7 days. A very clear common periodic daily pattern appears and is compared to non-spanish words

The reason that each language has its own fingerprint could be

- The fact that each language has its own geographic distribution, and thus a different time-zone distribution.

- The habits related to working hours, eating hours etc may differ from one community to another, and these habits may imply different distribution of tweeting times.

Can we check which of the above is more pertinent? Observe in Figure 4 the frequency of appearance of the words "une", "della", "der". These three words correspond to articles in French, Italian and German. We see that the periodic frequency pattern of the three words is very similar. These three languages correspond are mainly spoken mainly in Europ, and the time zone in which they are spoken is the same. It thus seems that the geographic location plays a major role so that similar geographic location indeed gives similar fingerprints.

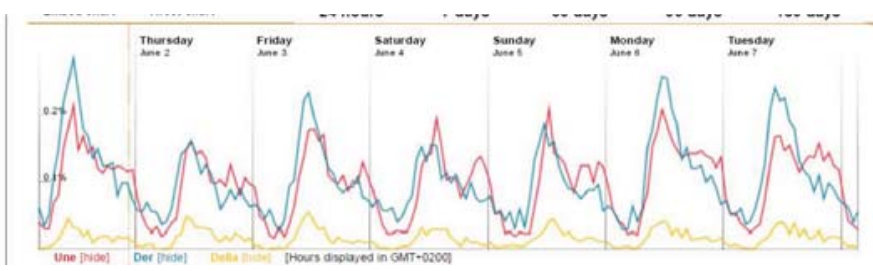

Fig. 4. The frequency of appearance of the words "une", "della", "der"

We next compare the Spanish word "Todas" with the French word "et". The Spanish one is seen to be shifted with respect to the French word by around 6 hours. For example its lowest activity during the day appears around 6 hours later than that of the French word. This suggests that most tweets in spanish originate in Latin America which has a time difference of 6 hours or more with respect to France.

\section{More DeTAILED GEO-LINGUISTIC FINGERPRINTS}

Daily periodograms can be made more selective so as to restrict to a subregion in which a language is spoken. As an example, we compare tweets with the Spanish words "computadora" and "ordenador". Both mean "computer", but the first is used in Latin America the second in Spain. The corresponding peroiodograms appear in Figure 5. We see that the term used in Spain has its minimal appearence around 8 hours before the Latin American one.

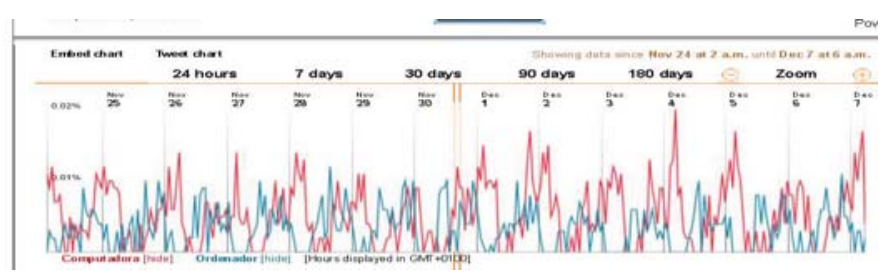

Fig. 5. The frequency of appearance of a word in Spanish from Latin America and that from Spain

We note that the average daily number of tweets in which "ordenador" appears is around $2 / 3$ the one corresponding to "computadoras". Does this suggest that the fraction of spanish tweets originatinng from Spain is close to that originating from Latin America? To answer this question, we may wish to compare also other words, or in contrast, to see how the relative frequencies behave in other contexts. When comparing the number of appearence of these words over the whole Internet, by using fightgoogle, we obtained (on Dec. 7, 2011) the figures: 4,580,000 for "computadora", and 7,150,000 for "ordenador".

Next we shall differentiate between the periodograms of the American versus the British versions of English. We do so by comparing the fraction of tweets containing "realize" (American version) and "realise" (British version) as a function of time, as is seen in Figure 6.

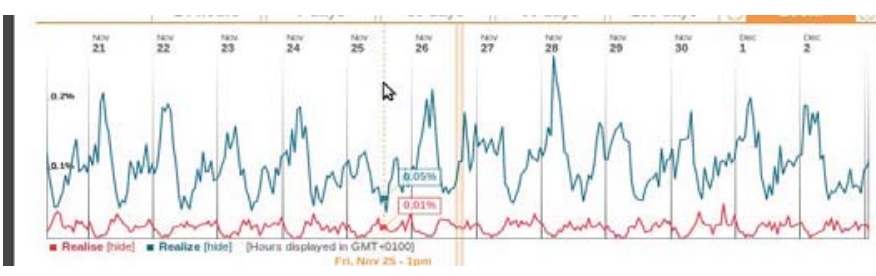

Fig. 6. The frequency of appearance of the words "realise" and "realize"

We see clearly that the minimum daily activity of the American word occurs around 6 hours later than the British one. 


\section{TWINGLISH AND OTHER LANGUAGES}

We focus in this section on some spellings or forms of writing words that are typical for social media [3], [4], [5]. We highlight some geographic aspects relalted to these.

"My son" in Spanish appears in Twitter often as "mijo" which is an abreviation of the two words "mi hijo". Figure 7 shows the daily pattern of the use of the word. All apearences of the word which we observed were indeed in Spanish. There is a clear inactivity period that corresponds to around $8 \mathrm{am}$ in French time. We conclde that the term "mijo" probably originates from the west part of Latin America. Similar behavior characterizes the word "porfa" whose periodogram is given in Figure 8. This is a way of shortening the word "please" in Spanish, which is written as "por favor".

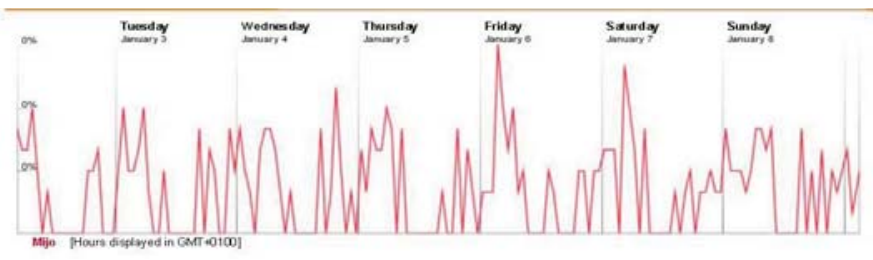

Fig. 7. The frequency of appearance of "mijo"

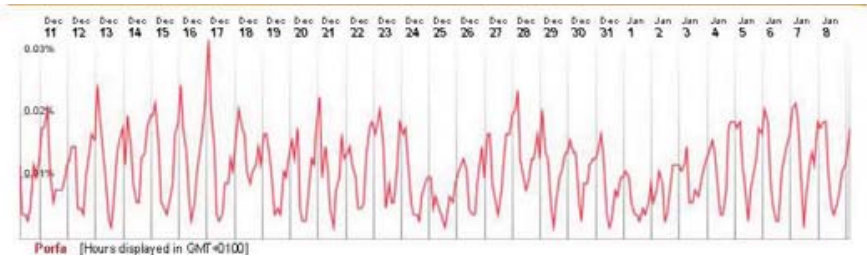

Fig. 8. The frequency of appearance of "porfa"

In contrast, the word "xk" which means in twitter-Spanish "because" or "why" [5] and is pronounced "porque" has no inactivity periods, see Figure 9. "xk" is much less localized and is probably used both in latin America and in Spain. Note that the translation of "por" using " $x$ " is due to the interpretation of $\mathrm{x}$ as multiplication, which is pronounced as "por".

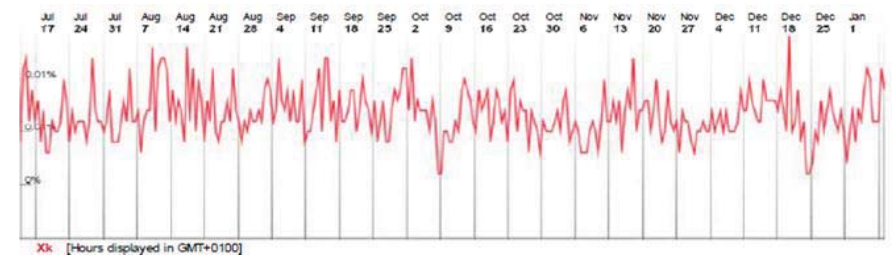

Fig. 9. The frequency of appearance of "xk"

We next observe the evolution of the word "xo".

The online urban dictionary http://www.urbandictionary.com/ says that $\mathrm{x}$ means kiss and o means hugs. xoxo then means "kisses and hugs". We found out that in Spanish "xo" is also used to say "pero" ("but" in English), where the explanation for the use of $x$ is as in $x k$.

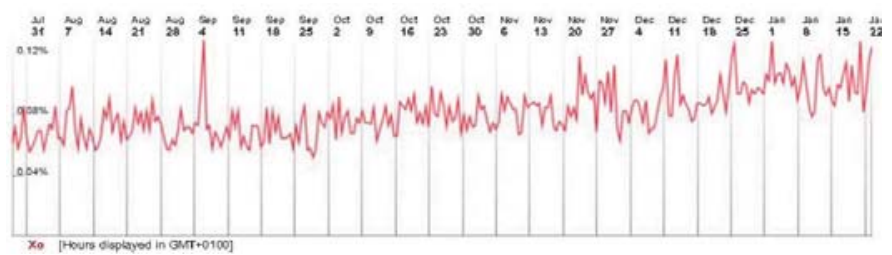

Fig. 10. The frequency of appearance of "xo"

In addition to spanish, $x k$ is also used in the same meaning in Italian (for the Italian word "perche" meaning "why"), see [6]. Note that the first vowel is pronounced different than in Spanish.

When working with trendistic, we can use the ratio between the maximum and minimum of the activity level during a day as a measure of its locality. We shall say that a term is well localized if this ratio is larger than 2 .

\section{The Spanish Word Porque}

We discuss in some more details the spelling we find in twitter for the words "porque" and "because".

We already mentionned the spelling "xk" for "porque". We found many other spellings. We list them along with the number of tweets in which they appear averaged over the six months period of beginning of Aug 2011 - end January 2012.

We tried also the following spellings: "porque" (0.5\%), "xq" $(0.07 \%)$, "porq" $(0.04 \%)$, "xk" $(0.012 \%)$. The frequency of their appearence in twitter is depicted in Figures 11 and 12.

Other spelling had too few occurrences and trendistics gave the message "There is too little data for a full chart so we are showing only recent activity". These spellings are "podque", "podq", "podk". They are obtained by replacing the "r" by "d" in the word "porque" and then, for the two last spellings, "que" is abreviated. Such a replacement is a typical childish way of speaking spanish, as many children have difficulties to pronounce the $r$ and replace it the by $d$.

We found no tweets with the spelling "xque". The spelling "pork" appears, but most tweets with this spelling correspond to the English word "pork".

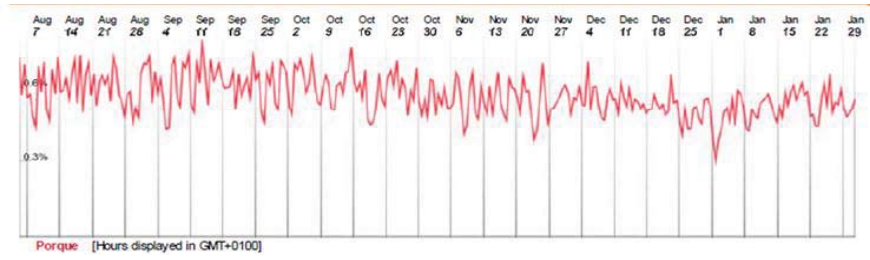

Fig. 11. The frequency of appearance of "porque"

\section{THE ENGLISH WORD "BECAUSE"}

The word "because" appears in twitter with a large number of variations. In fact, the total fraction of tweets in which this word appears in a new form and/or spelling is larger than that corresponding to the original word. This is illustrated in Table I which provides the most frequent variations of "because" along with the number of tweets in which they 


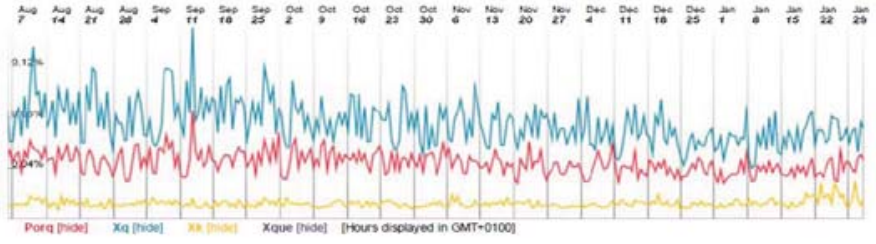

Fig. 12. The frequency of appearance of other spellings of "porque"

appear (averaged over 300 samples) along with the standard deviation. For reference, the average fraction of tweets in which the word "because" appears without changes is $0.824 \%$ of the tweets with a standard deviaition of $0.107 \%$.

Two short forms appear frequently in twitter: "cuz" (around $0.2 \%$ of tweets) and "coz" (around $0.02 \%$ of tweets). Such shortening of words are called "clipping" in linguistic research [4]. Twinglish thus allows us not only to recognize the word but also to hear it, and hence distinguish between the American and British accents. (Note that the opposite happened with the word "xk" which means "why" both in Italian and in Spanish, but is pronounced differently in the two languages.))

In Figure 13 we observe the periodograms of both. We see that "cuz" and "coz" have exactly the opposit activity profile: the minimum activity of "coz" are during night hours in Europe where as those of "cuz" are in night time in USA and Canada. The maximum daily activity of "coz" is during day time in Europe where as "cuz" has its maximum activity at day time in America. The periodogram of both words show very well localization: the ratio between the peak and the minimum activity is around 5 for both "cuz" and "coz".

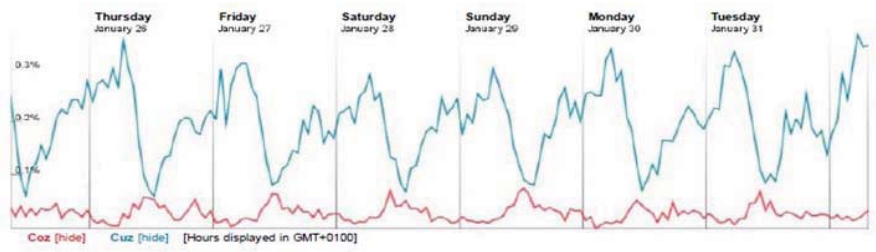

Fig. 13. The frequency of appearance of popular spellings of "because"

We also find the spelling "cus" and "cos" as seen in Figure 14-15. Again, the spelling is seen to correspond to the accent. The geo-linguistical finger print of "coz" is seen to be the same as "cos" (Fig 14). They both correspond to the UK where the second vower of "because" sounds like "o", as opposed to the American pronounciation that sounds like "u" which we find in "cuz" and "cus".

When comparing the two "American" spellings "cuz" and "cus", we see that there is a very clear preference to "cuz" where as the British seem quite indifferent between the two British spellings "cos" and "coz". The preference of the version with " $\mathrm{z}$ " in the America is in line with the fact that there have been already much before twitter differences between UK and USA with respect to the use of $s$ versus $z$.

Further shortning of "coz" and "cuz" by elliminating the vowel is possible but it did not seem appealling to Twitternauts. We have not found "because" written as "cs". It appearead however as "cz", four times less frequently than "coz". From its periodogram in Figure 16, "cz" is seen to be very localized and it corresponds to the same activity period as that of "coz". We conclude that the use of "cz" is restricted to Twitternauts from UK.

Two other spelling, "bcuz" and "becuz", appear with lower activity Their periodogram in Figure 17 shows activity periods that correspond to in America. We again have high degree of localisation. We did not find tweets with the spelling "bcz" or "bcos".

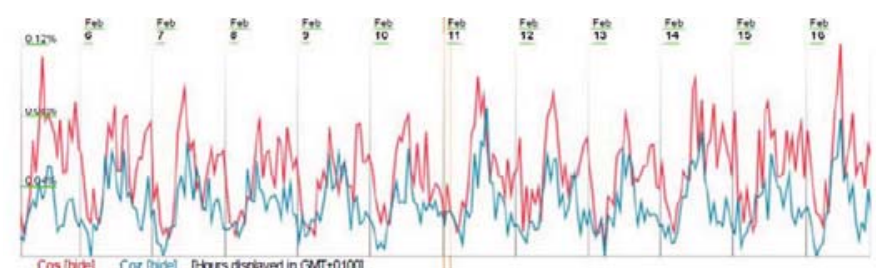

Fig. 14. The frequency of appearance of the spellings of "cos" and "coz" of "because"

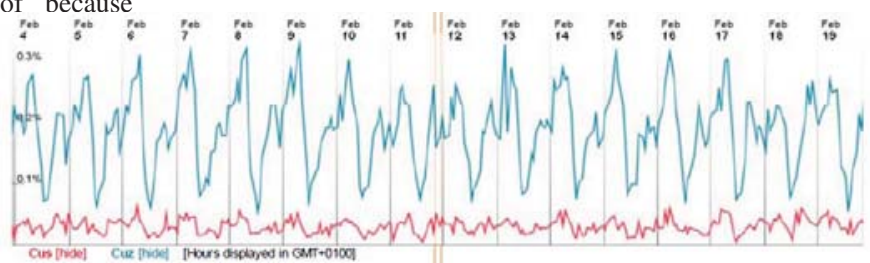

Fig. 15. The frequency of appearance of the spellings "cus" and "cuz" of "because"
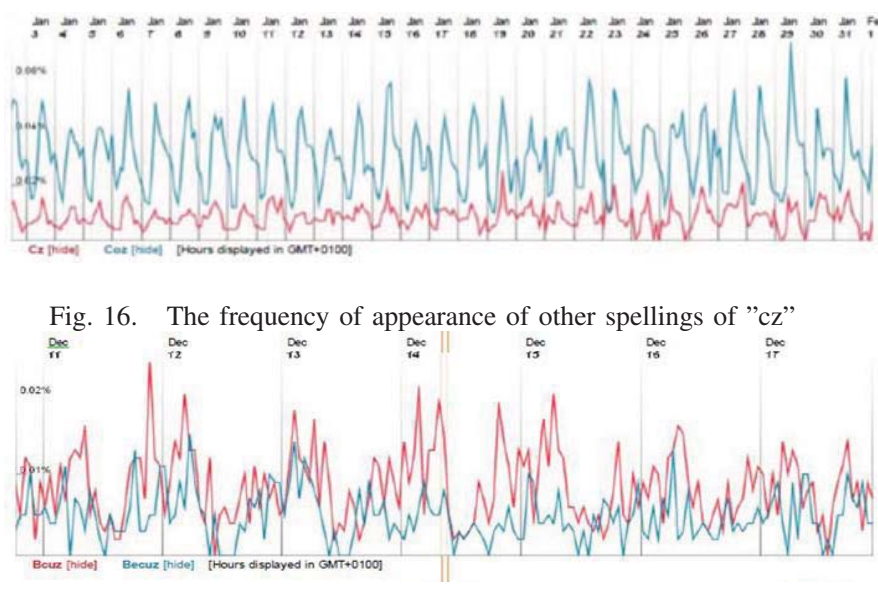

Fig. 17. The frequency of appearance of the spelling "bcuz" and "becuz"

Remark 1. Many of the spellings that we presented have other meanaings than because. For example, cos is also the mathematical function "cosine", "cause" usually means "reason" and is also a verb, "cz" is used in other context for the Czech Republic (it has also other meanings). Thanks to the snapshots of the contents of the tweets that trendistics provides, we were able to confirm that the above spellings are indeed used in twitter mainly in the sence of because. We shall later see that this is not the case in other electronic media. 


\begin{tabular}{|c|c|cc|cccccc|}
\hline Spelling: & because & bcuz & becuz & cause & cuz & cos & cus & coz & cz \\
\hline \% of tweets in & & & & & & & & & \\
which it appears: & 0.824 & 0.0156 & 0.0116 & 0.505 & 0.187 & 0.0490 & 0.0304 & 0.0135 & 0.0128 \\
Standard deviation & 0.107 & 0.00350 & 0.00355 & 0.0678 & 0.011 & 0.0141 & 0.064 & 0.011 & 0.00355 \\
\hline
\end{tabular}

TABLE I

THE MOST POPULLAR SPELLINGS OF "BECAUSE" IN TWITTER

\section{COMBINATION OF DIFFERENT SPELLINGS}

In view of the large number of different spelling of words, the natural question that arises is of what information is conveyed by a different spelling of a word. We already saw that the spelling can convey to which geo-linguistic group one belongs, e.g. whether the message is written by a British or an American English speaking person. The differentiation between these English versions that already existed since long ago has increased in tweeter, not only with respect to other media but also with respect to other social networks.

In this Section we examine other information and semantic content that spelling conveys. This has been motivated by a large number of messages in twitter in which we found various spellings of the same word in the same message. Is it intentional?

We focus on the word "because" in English and Spanish. Here are some examples of intentional use of different spellings or forms of the word.

We found different spellings appearing in the same sentence when comparing between two reasons for some action or some decision. Here are some examples that we found in tweets written in the end of March 2012 and the beginning of April.

1) 26 March, 2012: "laugh at u because ur different?? Laugh at them becuz they're the same!"

The example did not specify in what sense the one or the others are different or not. This is left to the imagination of the reader. My own prefered interpretation is that the difference is in the way of spelling "because", and hence the message advises one not to be too hurt if they laugh at you because you use a different spelling for writing "becuz", but rather to laugh at them becuz they all write becuz using the same spelling.

2) 26 March, 2012: if you haven't notice by now... i'm really bad at replying to ppl, not becuz im ignoring you, just because im a lazy person lol.

3) March 27th, 2012. BTW...that LOL was because of fun memories...not becuz of song that was tweeted by RT

4) 27 march, 2012: i cannot wait til spring break 2013, nt because of the trip but becuz on my way back ill be saying, this is my last time drivin to muncie :)

5) April 2nd, 2012: In class a professor called US a pure socialist country becuz it bails out all its companies, and Russia a capitalistic one because it wudnt

6) April 4th, 2012: U don't want to get to kno me becuz of something I did or somewhere I've been...u want to get to kno me just because of how I look..

The same structure appears also in Spanish, for example:
- 4th April, 2012: no llueve porque tu salgas del colegio, llueve xk son vacaciones y en vacaciones siempre llueve yo creo que es por joder!

which says - it is not raining because you got out of school, it rains because these are holidays ...

Another case in which we observe a tendency to alter the spellings of the word "because" in a sentence is when the sentence has a nested structure. For example

- April 4th, 2012: I isolate myself when I feel a certain type of way \& I'm trying to get it off my mind because I don't like for people to be down becuz of me

In Spanish, the word "porque" means both "because" as well as "why". Observing all occurrences of both in the week of 26 March - 4 April 2012, 8 out of 9 used "xk" for why and "porque" for "because", and only one case was the opposit. This suggests that new spellings can be used to transfer more information on words that looked the same in previous spellings.

\section{COMPARISONS WITH OTHER TYPES OF CONTENTS AND TOOLS}

In this Section we compare the freqency of occurrance of "because" in its different spellings obtained in Twitter to the frequency of its appearance in the World Wide Web. For the latter we used "google search". We repeated the same experiment restricting to those pages that fall into the category of "news" under google.

Our findings are summarized in the Table II.

The table presents the normalized popularity: at each row, we divided the corresponding number by the first one in that row. In that way we can compare the relative "popularity" of each form of "because" on different media.

The fractions of the versions of "because" that are obtained by shortening, "becuz" and "bcuz", are seen to be much higher it tweeter than those obtained in google and in google news. The number of appearances of each one of these two versions, divided by the number of times that "because" appears, is more than 500 times larger in twitter than over the whole Internet. It is more than 100 times larger in twitter than it is over news documents found by twitter.

This ratio in twitter is also larger that in the whole internet for the versions obtained by further clapping the word "because" (where the first cylable disappears) with one exception: the word "cause" which is used more on the Internet since the other meanings of the word "cause" appear more frequently there. This is also true to the finding over google news.

Two other exceptions occur with respect to google News: "cz" is very frequent there as it is used with the meaning of 


\begin{tabular}{|c|c|c|c|c|c|c|c|c|c|}
\hline Spelling: & because & bcuz & becuz & cause & $\mathrm{cuz}$ & $\cos$ & cus & $\mathrm{coz}$ & $\mathrm{cz}$ \\
\hline $\begin{array}{l}\text { Normalized popularity } \\
\text { trendistics }\end{array}$ & 100 & 2.00 & 1.41 & 61.3 & 22.7 & 5.94 & 3.69 & 1.64 & 1.55 \\
\hline Google & 100 & 0.00251538 & 0.00264231 & 26.8461538 & 0.8076923 & 1.0384615 & 0.0869231 & 0.2115385 & 0.9769231 \\
\hline Google News & 100 & 0.0171975 & 0.0140127 & 122.2929936 & 0.4356688 & 11.1464968 & 0.656051 & 7.1974522 & 48.4713376 \\
\hline
\end{tabular}

TABLE II

NORMALIZED POPULARITY OF VARIANTS OF BECAUSE IN \%

the Czech Republic. The spelling "cos" also has many other uses that appear in google News.

\section{COnClusions}

This paper contributes to identifying and understanding some of transformations in spelling and use of words over twitter. This includes a geo-linguistic analysis that allows one to track different types of transformations in different communities that have a common language in common. Among the many examples presented here, we have studied in more detailed the transformations of the word "because", both in English as well as in Spanish. We saw that some common form of using two different spellings in the same sentence has emmerged both in Twinglish as well as in twitter-Spanish. We managed to differentiate between the locations of various versions of "because" in English.

The creation of new spelling and forms of words in twitter is often explained by the advantages in writing shorter words: both the character limitation in twitter as well as the fact that many tweets are sent from cellular phones whose small keyboard is not as confortable as that of a laptop.

In the creation process of new spellings, alpha-numerical symbols often replace cylables according to

(i) the phonetic sound that they are associated with. Examples are 3Q which is used in Chinese as for "thank you". We call this an "audio association".

(ii) the graphic form that they have. That symbol " 3 " is a "graphic association" of a heart or lips and is used for expressing affection. The number 7 has a form similar to that of the letter "cha" in Arabic and is thus used as such when an arabic keyboard is not available.

(iii) Composition of associations: we saw that "xk" means "because" The "x" is pronounced "por" through a two step association: first a graphical association is used to transform "x" to "multiply", and then the audio association of "multiply", which is "por" in Spanish, is used.

The audio associations are often innexact. Here are some examples.

- The letter "k" is pronounced as "ka" in Spanish so that "xk" sounds as "porqua" where as it is used in the meaning of "because" in Spanish, which sounds like "porque".

- "k2" sounds as "KaDeu" in French and means a "present"; the pronounciation of "present" in French is, however, "KaDo".

- The word "your" is often shortened to "yo".

It is not a surprise that there is a big tolerancne to such imprecisions, as we know of natural languages in which the vowels, altogether, do not appear in the written version (e.g. Hebrew or Arabic). Yet, although we see vowels appear often in an imprecise way in Spanish, English and French, the Twitterenauts do not seem eager to drop them completely (as we saw in the shortning of "because").

We saw that Twitternauts often convay the accents they use. This was the case of the word "because" whose twitter spelling spelling "cuz", "becuz" or "bcuz" suggest the USA accent whereas its spelling "coz" suggests the British one. We showed that this classification is confirmed with a high degree of localization obtained using the periodograms.

Further audible features of words appeared, e.g. in replacing the "r"s by "d"s in Spanish, as we saw in the word "porque". This feature also occurs in English, where the sound "th" in words such as "the", "this" and "that" is sometimes pronounceed as a "d". We illustrate this in Figure 18.

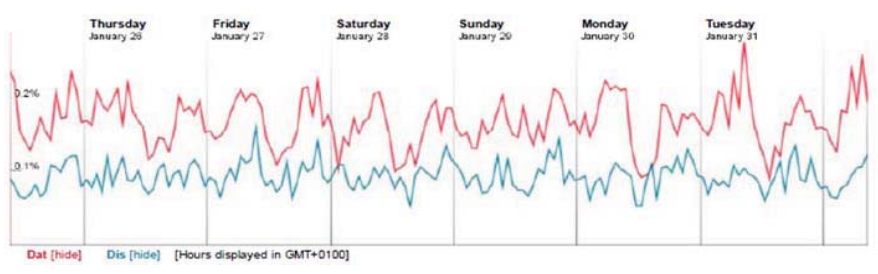

Fig. 18. The words "this" and "that" spelled as "dis" and "dat"

\section{Acknowledgement}

The work was supported by the Agorantic S.F.R. (Structure Fédérative de Recherche) on "Sciences and Technologies of Cultures and Digital Societies" University of Avignon (UAPV).

\section{REFERENCES}

[1] O'Connor, B.; Balasubramanyan, R.; Routledge, B. R.; and Smith, N. A. 2010. From tweets to polls: Linking text sentiment to public opinion time series. In Proc. 4th Intl. AAAI Conf. on Weblogs and Social Media (ICWSM).

[2] Tumasjan, A.; Sprenger, T. O.; Sandner, P. G.; and Welpe, I. M. 2010. Predicting Elections with Twitter: What 140 Characters Reveal about Political Sentiment. In Proc. 4th Intl. AAAI Conf. on Weblogs and Social Media (ICWSM)

[3] Stephan Gouws, Donald Metzler, Congxing Cai and Eduard Hovy, "Contextual Bearing on Linguistic Variation in Social Media", Proceedings of the Workshop on Language in Social Media, June 23, 2011.

[4] Hong-mei Sun, "A Study of the Features of Internet English from the Linguistic Perspective", Studies in Literature and Language, Vol. 1, No. 7, 2010, pp. 98-103.

[5] Isabel Gretel María Eres Fernández and Paulo Augusto Almeida Seemann, "A study on language changes of written spanish in internet chats" Trab. linguist. apl. vol.48 no.1 Campinas Jan./June 2009

[6] Zoe Rimay, "Cybercultural Communication", Budapest Univ. of Technology and Economics, Faculty of Economic and Social Sciences. Report. 